\title{
Surfaces
}

\section{FÉMINISME ET WOMEN'S STUDIES. VIEILLES HISTOIRES}

\section{Rachel Bowlby}

Volume 3, 1993

URI : https://id.erudit.org/iderudit/1065099ar

DOI : https://doi.org/10.7202/1065099ar

Aller au sommaire du numéro

\section{Éditeur(s)}

Les Presses de l’Université de Montréal

ISSN

1188-2492 (imprimé)

1200-5320 (numérique)

Découvrir la revue

Citer cet article

Bowlby, R. (1993). FÉMINISME ET WOMEN'S STUDIES. VIEILLES HISTOIRES.

Surfaces, 3. https://doi.org/10.7202/1065099ar
Résumé de l'article

L'auteure tente une reconstruction de l'histoire des women's studies et de la dynamique qui les relie au féminisme. Évoquant les diverses polarités qui ont structuré les conditions de leur interaction, elle décrit l'évolution du féminisme anglo-américain, du féminisme français et du French feminism en faisant intervenir les notions de marginalité et d'institutionnalisation, les théories de Kristeva, de Cixous et d'Irigaray, la déconstruction et l'" essentialism » et, dans une visée plus polémique, la " domestication ». Pour illustrer la thèse que les enjeux du débat entourant le rôle des women's studies au sein du féminisme n’ont pas changé, Bowlby offre une critique déconstructrice du récent livre de Judith Butler, Gender Trouble.
Copyright (C) Rachel Bowlby, 1993

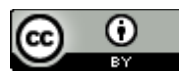

Ce document est protégé par la loi sur le droit d'auteur. L’utilisation des services d'Érudit (y compris la reproduction) est assujettie à sa politique d'utilisation que vous pouvez consulter en ligne.

https://apropos.erudit.org/fr/usagers/politique-dutilisation/ 


\section{FÉMINISME ET WOMEN'S STUDIES}

\section{VIEILLES HISTOIRES}

Rachel Bowlby

\section{RÉSUMÉ}

L'auteure tente une reconstruction de l'histoire des women's studies et de la dynamique qui les relie au féminisme. Évoquant les diverses polarités qui ont structuré les conditions de leur interaction, elle décrit l'évolution du féminisme anglo-américain, du féminisme français et du French feminism en faisant intervenir les notions de marginalité et d'institutionnalisation, les théories de Kristeva, de Cixous et d'Irigaray, la déconstruction et $l '<<$ essentialism $>>$ et, dans une visée plus polémique, la $<<$ domestication $>>$. Pour illustrer la thèse que les enjeux du débat entourant le rôle des women's studies au sein du féminisme n'ont pas changé, Bowlby offre une critique déconstructrice du récent livre de Judith Butler, Gender Trouble.

\footnotetext{
ABSTRACT

An attempt to reconstruct the history of women's studies and of the dynamics of its relationship to feminism through the analysis of the polarities that have structured the conditions of interaction between the two "sister entities." Various categories and distinctions are brought up such as marginality and institutionalization; Anglo-american feminism, French feminism and "French feminism;" the theories of Kristeva, Cixous and Irigaray; deconstruction and essentialism, and more polemically, "domestication." By way of showing that the stakes in the debate upon the role of women's studies in feminism remain unchanged, Bowlby offers a deconstructive critique of Judith Butler's recent Gender Trouble.
} 
Parler du féminisme et des women's studies, c'est parler, semble-t-il, de deux êtres, ou de deux entités, qui ont beaucoup en commun, qui sont liés comme deux soeurs inséparables. Oui, mais... du moment où on les met ensemble, ils (ou elles) se disputent; c'est le féminisme qui gagne, tandis que les pauvres women's studies sont reléguées dans la position conservatrice, symbole de l'assimilation ou de la normalisation d'un mouvement qui se voulait radical et marginal. Car les women's studies font partie de l'institution universitaire; le féminisme, en s'identifiant à elles, rejoint le pouvoir, sinon le patriarcat en personne.

Mais qu'est qu'on fait là-dedans, à l'intérieur de ces nouveaux départements de women's studies? Là aussi, la critique n'hésite pas. C'est très simple, diton (dit le 'on' qui s'identifie avec un féminisme authentique, non contaminé): les women's studies, c'est une célébration naïve des femmes et de leur histoire qui ne remet pas en question le statut de l'objet étudié. Les femmes ont toujours été exclues de l'histoire 'patriarcale', qui ne s'intéresse qu'aux hommes et aux faits valorisés par les hommes. Le projet des 'women's studies' serait donc d'offrir les moyens de réparer cet état de choses. L'addition des women's studies comblerait un manque qu'on vient de percevoir; elle ne changerait rien à la structure générale des connaissances.

En tant que discipline académique, les women's studies s'ajoutent sans difficulté à la liste des matières ou des 'majors' sans rien changer, selon les critiques, aux pratiques ou aux présupposés des autres disciplines. Elles s'intègrent à l'institution et aux dispositifs épistémologiques existants. Ce serait un peu comme si on ajoutait un état supplémentaire à l'union des États-Unis. Comme dans le cas de Hawaï: ça peut avoir l'air un peu différent, un peu exotique, même un peu lointain par rapport aux autres disciplines, mais ça s'intègre tout de même. Les petites frontières des états restent les mêmes et le grand ensemble imaginaire de l'Amérique se trouve renforcé. Il y a du nouveau à l'horizon, mais ce nouveau n'est pas menaçant; au contraire, il sert à faire voir que le grand état est assez fort pour s'imposer comme norme à la fois politique, juridique, économique, et imaginaire (l'idée de la nation américaine).

Les Women's Studies, bien sûr, se veulent féministes, mais pour leurs opposants, ou leurs opposantes, ce qu'elles représentent - -- ou ce qu'elles représentaient au début (j'ai l'impression que cela a changé) --, c'était un féminisme bien limité, dans tous les sens du terme (enfermé dans son propre petit département, incapable de se faire vraiment radical). Selon cet autre féminisme qui, aux États-Unis du moins et parfois en Angleterre, s'est souvent représenté comme 'français' (il y a là une ironie sur laquelle je 
reviendrai), le féminisme cesse d'être féministe du moment où il perd son statut marginal d"outsider' par rapport à toutes les disciplines, ou par rapport aux institutions. Si le féminisme fait partie de l'institution, s'il s'incorpore comme une connaissance parmi les autres, il ne modifie plus rien à l'ensemble; il devient lui-même une institution et cesse d'être un mouvement -- mouvement politique, mouvement théorique, mouvement tout court au lieu de fixité.

On se trouve plongé d'emblée dans toutes sortes de difficultés linguistiques. Les women's studies, ça ne se traduit pas. Qu'est-ce que ça veut dire, au juste? Qu'on étudie les femmes? Qu'il s'agit des études pour les femmes? Que c'est ce que les femmes étudient ou devraient étudier? En anglais, il n'existe pas d'adjectif convenable -- pas d'équivalent, par exemple, à 'AfroAmerican Studies' ou à 'lesbian and gay studies'. 'Womanly Studies', ça poserait certains problèmes. On a donc recouru à cette lourde formulation de 'women's studies', et il paraît que le nom, malgré ses ambiguïtés grammaticales, n'a fait que renforcer l'idée d'une solidité immuable, que ce soit du côté de l'objet ou du sujet (étudier les femmes, études des femmes ou pour les femmes).

On aurait pu dire 'feminist studies': c'est le titre d'une revue américaine. C'est le titre aussi, plus ou moins, d'un cours enseigné à Sussex, l'université où j'enseigne en Angleterre: 'Studies in Feminism'. Mais cette alternative n'a pas connu de succès aux États-Unis. Pourquoi? On peut se demander si ce n'était pas pour la raison même qui fait qu'on accuse les women's studies d'être limitées. Car on peut dire que le nom de 'feminist studies' exclut d'emblée les questions de principe ou de base, parce qu'il se propose déjà un but politique connu d'avance. Ou qu'il n'implique que l'étude de l'histoire du féminisme, en rejetant tout ce qui pourrait concerner les femmes ou la féminité, en général, sans parler d'autres possibilités.

Je crois que c'est en ces termes que le débat autour du nom du nouveau cours a eu lieu à Sussex (c'était déjà une vieille histoire quand j'y suis arrivée en 1984). D'une part, on disait que la dénomination 'Women's Studies' faisait empiriciste, non-théorique, et que c'était une étiquette figée et surtout figeante: femmes, voici votre place! D'autre part, on disait que 'feminist studies' ou 'studies in feminism' constituaient des appellations tout aussi étroites, renvoyant exclusivement à la politique, ou à une politique déjà établie qui ne serait plus à mettre en question.

Depuis, il y a eu à plusieurs reprises des discussions autour de la même question sous une forme un peu différente: devrait-on ou pas offrir une maîtrise nommée 'women's studies"'? Les deux arguments contre cette proposition n'ont aucun rapport l'un avec l'autre. D'abord, qu'est-ce qu'on enseignerait comme 'cours de base': l'histoire des femmes (mais quelle 
histoire)? l'histoire de la pensée sur les femmes? l'histoire de la pensée des femmes et des théories féministes? Ensuite, tous les cours devraient être des cours féministes ou poser des questions féministes. Instituer un lieu distinct désigné par 'féminisme', plus encore un lieu dit 'women's studies', aurait l'effet de le mettre à part, dans deux sens: premièrement, en impliquant que le féminisme (et les women's studies) sont sans rapport avec les autres études; deuxièmement, en les marginalisant de nouveau (dans ce cas, la marginalité devient une valeur négative signifiant 'sans influence', dépourvus de pouvoir critique ou autre).

Si j'ai choisi l'état, ou les états américains, comme analogie pour parler de l'entrée des women's studies dans la gamme des disciplines universitaires, ce n'est sans doute pas un hasard. D'abord, très simplement, parce que les women's studies sont surtout un phénomène américain. Historiquement, le nom est une invention américaine -- ou bien, ce qui revient au même, on croit que c'est une invention américaine. En France, je le dis en passant, l'équivalent des women's studies n'existe presque pas dans les institutions, que je sache, bien que l'expression 'études féminines' soit employée (l'adjectif évite les problèmes du nom ' women' en anglais). Il existe un centre d'études féminines à Saint-Denis, ex-Vincennes, dans le cadre des groupes de recherches intra-européens, mais je crois qu'il est presque unique. Cette différence culturelle mériterait d'être étudiée. En Angleterre (je ne peux pas parler des autres pays anglophones), la notion de 'women's studies' se lie à son américanicité (comme dirait Barthes), ce qui a infléchi différemment les débats qu'a connus ce pays. On est revenu, sous une autre forme, à la question de la marginalité.

De l'autre côté de l'Atlantique, on a tendance à percevoir l'université américaine comme une organisation qui ne diffère pas des entreprises capitalistes. On dénonce les pratiques d'achat et de vente -- l'inflation des salaires des professeurs, le 'star system' qui fait de l'université un petit Hollywood manqué: on aperçoit un article sur le colloque MLA dans le New York Times magazine, on dit que Stanley Fish gagne cent cinquante mille dollars (c'est toujours lui qui est cité), etc. Quant aux cours, on parle de supermarché: on évoque ces étudiants qui choisissent n'importent quoi, uniquement pour le plaisir, qui font du 'shopping around' au début du trimestre pour voir s'ils aiment tel professeur ou pas. Comme toujours, les Britanniques (et les Français aussi, mais pas tout à fait de la même façon) parviennent sans difficulté à voir dans l'Amérique le symbole d'une vulgarité capitaliste, et surtout commerciale, dont il sont eux-mêmes, bien sûr, exempts.

Dans ce contexte, les women's studies sont condamnées non seulement à cause d'une simplicité intellectuelle qui leur est attribuée sans autres précautions, mais aussi parce qu'elles font partie de ce système d'options qui mise sur le choix du consommateur où la nouveauté et l'innovation sont perçues comme un nouveau produit, une nouvelle marque de commerce à 
vendre. Le féminisme ne s'achète pas, il ne se vend pas: il n'ira donc pas au marché.

Il est intéressant de noter ici une opposition bien traditionnelle entre l'authentique et le commercial, selon laquelle la pensée pure serait hors marché et, en deçà de ce présupposé, que la pensée, quelque part, serait la pensée pure. Si on veut opposer pensée pure et pensée impure (ou contaminée), la figure du marché est toujours disponible -- mot lui-même inséparable, il me semble, d'une logique de la vente et de l'achat. Car le marché offre l'image d'un pouvoir énorme, irrésistible -- le capitalisme luimême, en personne -- et aussi une image de nullité, de trivialité: la marchandise ou le nouveau produit peuvent être n'importe quoi, pourvu qu'ils paraissent comme 'neufs', ou comme différents des autres: mais cette nouveauté et cette différence seront toujours superficielles, sans réalité.

En Angleterre, très simplement, il n'y a pas d'argent pour fonder de nouveaux programmes académiques ou des centres de recheches, que ce soit pour les women's studies ou n'importe quelle autre discipline ou programme interdisciplinaire. Surtout au début des années 80, les coupures budgétaires effectuées par le gouvernement de Madame Thatcher dans le domaine de l'éducation universitaire ('the cuts', comme on disait) faisait qu'il n'y avait presque pas de nouveau postes, et même presque pas de postes de remplacement dans les départements établis. Donc la critique des women's studies américaines n'allait pas sans un élément de ressentiment, du genre 'les raisins trop verts': là-bas, ils ont de l'argent, ils ont des moyens; mais nous, nous ne voulons pas nous compromettre de cette façon-là, en faisant de notre féminisme révolutionnaire une discipline conformiste, bien élevée et bien adaptée aux normes du comportement institutionnel.

Mais il y a une autre polarité qui joue très souvent dans l'opposition aux women's studies, surtout dans le domaine de la critique littéraire: je parle de la distinction souvent invoquée entre le féminisme 'anglo-américain' et le féminisme 'français'. Bien qu'étant géographique, ou peut-être linguistique en apparence, en fait cette opposition ne recouvre aucune différence réelle entre les anglophones et les francophones, encore moins (on vient de le voir) entre la France, d'une part, et un bloc, d'autre part, qui comprendrait les États Unis et l'Angleterre.

Commençons par les mots (il n'y a que les mots, après tout). Ce qui s'est appelé féminisme, en France, ne correspond pas à ce qui a été appelé féminisme français aux États-Unis ou en Angleterre. Le féminisme appelé féminisme en France représente ce que Julia Kristeva, dans son essai intitulé 'Le temps des femmes', a appelé une logique de l'homologation.[1] (Il est significatif, pour la problématique dont je parle, que cet essai, souvent republié en anglais, soit introuvable en français.) Selon cette logique, les 
femmes auraient demandé accès au droit de participation à la démocratie. Comme d'autres groupes exclus des démocraties modernes, selon des critères de propriété, d'âge ou de race, les femmes ont revendiqué une égalité qui les ferait reconnaître en tant que sujets politiques, juridiques, ou économiques (le droit au travail aux mêmes termes et au même salaire que les hommes).

Pour Kristeva, ce féminisme-là n'est pas à rejeter, loin de là, mais il ne représente qu'une première étape. La revendication de l'égalité implique à la fois la neutralité de l'espace, pour lequel on veut gagner l'entrée, et la possibilité de comparer les femmes aux autres groupes qui pourraient demander ce même accès. Mais pour Kristeva, cet espace n'est jamais neutre: il est masculin. La différence sexuelle est très précisément incommensurable avec les autres formes ou catégories de différenciation. Elle est fondamentale: première des différences, à la fois sur le plan du développement individuel et sur le plan ontologique. Mais ceci n'implique pas qu'on devrait recourir à l'idée d'une différence sexuelle déjà donnée, que ce soit par la nature ou par la culture: c'était la deuxième étape. Par l'utilisation d'un modèle psychanalytique, Kristeva est plutôt amenée à souligner, d'abord, le manque de correspondance entre les termes de 'femmes' et de 'féminité', ou d'hommes' et de 'masculinité'; puis, le manque de symétrie entre le féminin et le masculin, catégories qui ne sont pas nées égales, même si elles pourraient le devenir.

La troisième étape -- après la revendication de l'égalité, et suite à la contrerevendication d'une différence nette entre les hommes et les femmes -renoncerait à la lutte collective au profit des recherches menées au niveau individuel -- par la voie de la psychanalyse, ou de la maternité, ou des expériences esthétiques, par exemple -- où chaque femme aurait la possibilité d'explorer et de développer les déterminations de sa propre subjectivité.

Là, il ne s'agit plus de féminisme, français ou autre, selon les termes de Kristeva. Il s'agirait, peut-être, d'une sorte de post-féminisme, terme souvent utilisé en France pendant les années 80. Néanmoins, ce que Kristeva n'appelle pas le féminisme fait partie de ce qu'on appelle ailleurs le féminisme français; et ce qu'elle appelle féminisme serait plutôt ce qui est dévalorisé par les partisanes non-françaises du 'French feminism'. La logique n'est pas très différente: dans les deux cas, l'existence et la nécessité de la fameuse première étape, ou 'first wave feminism', sont présupposées. Mais la différence consiste, pour les non-Françaises, à nommer français ce genre de féminisme attribué aux Françaises, mais que ces dernières ne nomment pas féminisme. 
Les théories de Cixous et d'Irigaray ne sont pas tout-à-fait comparables à celles de Kristeva (bien qu'il y ait eu un moment, au tournant des années 80, où on avait tendance à mettre sur le même plan -- à homologuer -- tout ce qui avait été traduit de ces trois auteurs, au nom d'un certain 'French feminism'). En particulier, Irigaray et Cixous se sont moins intéressées à l'utilisation qu'à la déconstruction de la psychanalyse, qu'elles ont interprétée à la fois comme un exemple et une critique de la tradition phallocentrique occidentale (je parle au passé, parce que les textes qui ont eu le plus d'influence dans les pays anglophones -- le Spéculum d'Irigaray, $<<$ Le rire de la Méduse >> de Cixous et la partie de La jeune née qu'elle a écrite avec Catherine Clément -- datent tous les trois du début-milieu des années 70, et qu'il existe un décalage non sans importance entre les dates de sortie en France et celles des traductions anglaises). Je ne veux pas non plus assimiler Cixous à Irigaray. Ce qu'elles ont en commun cependant (également avec Kristeva), c'est d'avoir proposé une théorie générale de l'histoire de la pensée occidentale qui pose comme constitutive et fondamentale la différence sexuelle dominée par le pôle masculin: en un mot, que la pensée occidentale est phallogocentrique de part en part.

Au début des années 80, à l'époque où le French feminism avait encore l'air de quelque chose de nouveau et de radical, on avait tendance, aux ÉtatsUnis et en Angleterre, à le critiquer en disant qu'il était, au fond, essentialist. Je ne traduis pas ce mot, parce qu'il me semble qu'il n'a pas d'équivalent en français. Bien qu'on ait adressé des critiques comparables, en français, à l'égard des femmes appelées en anglais 'French feminists' -- je pense, par exemple, aux articles de la revue Questions Féministes --, le mot essentialist ou essentialiste, si vous préférez, n'est pas employé.

Je ne veux pas entrer ici dans les débats -- assez complexes et parfois très ennuyeux -- portant sur la signification exacte qui devrait être attachée à ce mot, avec la prétention philosophique et ce ton de condescendance un peu irascible qui semble vouloir dire aux féministes qu'on accuse d'essentialisme: <<Vous mettez trop de parfum!>> En gros, en les accusant d'essentialism, on reprochait aux féministes françaises de dire quelque chose du genre: <<la féminité, propre aux femmes, a toujours été refoulée ou supprimée par la société et les discours phallocentriques; il faut donc la libérer, ce qui bouleversera, en même temps, les structures de la langue et de la pensée et, a fortiori, les formes sociales, elles-mêmes structurées selon ce que la pensée rend imaginable $>>$. Se baser sur cette structure-là, selon les critiques, ne changerait pas grand chose: d'abord, parce qu'on n'opère qu'un simple renversement de valeurs, sans rien changer aux formes; ensuite parce que la nature de la féminité, qu'elle soit ou non considérée comme propriété exclusive des femmes, n'est pas mise en question.

Au début des années 80, il y eut toute une série d'articles en anglais qui disaient, plus ou moins: c'est très intéressant, très différent, ce féminisme 
français; mais finalement, ça retombe dans l'essentialism. À l'époque, il s'agissait d'une critique meurtrière. Une ironie supplémentaire est à noter ici: ces critiques se réclamaient souvent des maîtres-à-penser français - -Lacan, Derrida; elles se voulaient donc plus françaises, c'est-à-dire plus théoriques, que les Françaises elles-mêmes.

Je simplifie, mais c'est aussi l'effet rétroactif. Quand on essaie de reconstruire l'histoire, surtout l'histoire d'une pensée, on simplifie et les changements ont l'air d'avoir été inévitables: comme si les enjeux s'étaient présentés sous la même forme à ce moment-là que maintenant où ils donnent l'impression d'être des questions dépassées ou des erreurs corrigées.

Ce n'est pas que la question de l'essentialism soit elle-même réglée. Mais elle ne constitue plus une accusation portée contre des textes réunis sous l'étiquette de 'French feminism'. On a maintenant tendance à y voir plus de complexité, voire la complexité même. On distingue désormais différents écrivains, alors qu'on les assimilait auparavent sous la seule rubrique du féminisme français. Les différends se sont modifiés. Car autour du milieu des années 80 , une nouvelle opposition s'est développée dans laquelle 'French feminism' a joué le rôle du provocateur -- le marginal, le subversif -au lieu de représenter la stabilité d'une féminité essentielle, déjà donnée.

Cette opposition nouvelle oppose au féminisme français le féminisme dit 'anglo-américain'. La solidité et la fixité, ici, sont du côté des AngloAméricaines (rapport évident avec l'opposition feminism/women's studies): pauvres lourdes femmes, encombrées qu'elles sont par leur croyance naïve en l'existence des femmes (pas forcément l'essence des femmes, on va le voir) et leur croyance bien trop fidèle en l'existence d'un patriarcat dont l'entreprise consisterait à leur refuser l'accès aux droits et aux libertés dont jouissent déjà les hommes. Ce qu'on voit maintenant du côté des Françaises, c'est au contraire une complexité et une sophistication de la pensée qui vise à garder ouvertes les possibilités d'un changement global dans les façons de faire et d'être (mais il faut concéder que cette division temporelle n'est qu'une reconstruction faite après-coup: le rejet, au début, n'était ni général et ne faisait pas toujours au nom de l'essentialism.).

Ici aussi, on revient à une tradition bien établie où tout ce qui est du côté anglo-américain est censé être naïf, simple, 'common-sense', pragmatique; tandis que tout ce qui est du côté français est censément élégant, original, philosophique au sens propre (c'est-à-dire au sens propre à ceux qui se servent de ce type d'opposition). Bien sûr, cette opposition est toujours présentée au bénéfice de ce qui est nommé français (qui a donc gagné d'avance). Mais ce qu'il faut dire aussi, c'est que selon les présupposés théoriques des adhérentes du féminisme français, cette forme d'opposition binaire correspond à quelque chose de bien simple: déjà hiérarchisée, 
l'opposition n'en est pas une, parce qu'elle n'utilise les catégories négatives associées à l'anglo-américain que pour mettre en valeur les termes positifs associés au français. La bataille a déjà eu lieu, au moment même où elle vient à être désignée.

Je ne dis rien des différences entre ce qui est impliqué par cette opposition, d'une part, et la réalité du féminisme en France ou en 'Anglo-Amérique', de l'autre. On ne peut pas faire une opposition entre des noms et des réalités, sans tomber dans le même type de simplification pré-structuraliste que je viens d'identifier au sujet de l'opposition entre le féminisme français et le féminisme anglo-américain.: à savoir, quand on pose une théorie où le langage viendrait nommer une réalité déjà donnée, structurée d'avance. Tel mot, telle phrase, seront jugés selon leur exactitude par rapport à ce dont ils parlent: c'est un critère d'adéquation où le rôle du langage n'est que secondaire, ancillaire, non structurant.

Si on regarde de près (ou même de loin) l'opposition entre féminisme français et féminisme anglo-américain, on voit tout de suite que, du point de vue géographique, elle ne correspond à rien. Par exemple, celles qui sont désignées comme les représentantes du féminisme français, on l'a déjà vu, ne se nomment pas elles-mêmes féministes. Si on coupe en deux ce fameux 'anglo-américain', on voit que ce qui se passe de l'autre côté de la Manche n'a pas beaucoup en commun avec ce qui se passe outre-Atlantique; que cela a plus en commun, peut-être, avec ce qui se passe en France. De la même manière, on pourrait trouver en France des équivalents au féminisme dit anglo-américain, où on lutte pour l'égalité des femmes dans tous les domaines de la vie sociale, et où le statut de la différence sexuelle n'est pas mis en question

De même, si on examine l'opposition français/anglo-américain du point de vue linguistique -- féminisme francophone contre féminisme anglophone --, on constate également qu'elle ne tient pas. Car les textes à tendance 'française' sont souvent écrits en anglais, la plupart par des professeurs de lettres françaises ou de littérature comparée. L'idée du féminisme français, comme on a pu le voir, est une idée anglo-américaine, mais qui a été inventée pour refuser le féminisme anglo-américain qui, selon ce partage, comprend tout ce qu'on veut rejeter comme simple, naïf, 'première étape', et ainsi de suite. La confluence anglo-américaine dépend, pourrait-on dire, d'une simple coïncidence de langue: malgré de grandes différences dans les traditions intellectuelles, on attribue à l'Angleterre et aux États-Unis des ressemblances de famille -- le 'rapport spécial' dont parlent les politiques -qui n'existent pas.

Néanmoins, l'histoire ne s'arrête pas là, en disant: bien, on va corriger les erreurs. L'important, c'est que cette opposition ait pu 'prendre', ait pu se 
faire une histoire (le fait que j'en parle aujourd'hui en constitue un témoignage supplémentaire). Dénoncer l'opposition au nom d'une réalité qu'elle cache, ce serait une fois de plus oublier ou méconnaître les effets producteurs des discours. Ça n'implique pas qu'on ne devrait pas critiquer telles oppositions, mais plutôt qu'on devrait s'interroger sur leur conditions de signification -- linguistiques, culturelles, ou autres: sur la façon dont elles ont pu prendre un sens, ou contribuer à l'élaboration de questions.

Je crois qu'on ne parle plus maintenant avec autant de bruit ou de conviction d'une quelconque opposition entre le féminisme français et le féminisme anglo-américain -- ni, en fait, d'une opposition entre le féminisme et les women's studies. Les enjeux du débat, si c'en est un, se sont déplacés. Il faut souligner, de plus, que le 'on' que je ne cesse d'invoquer est une pure fabrication: il traduit un 'we' qui ne parle pas au nom de toutes les féministes anglophones, loin de là. Encore une fois, l'histoire que je décris ou que je raconte est une histoire qui se déroule 'en anglais', bien qu'elle s'appuie sur des textes français.

Un peu plus loin, je vais m'engager dans ce qui me paraît être une continuation, une prochaine étape peut-être, de ce questionnement. Pour le moment, je voudrais faire la transition, ou plutôt la simuler, en m'arrêtant sur un thème qui demeure caché dans les textes qui portent sur cette histoire: je veux dire la domestication.

En parlant non seulement de la théorie féministe, mais aussi de la théorie en général, en particulier de la déconstruction, on fait souvent appel, sans l'expliciter, à la notion de domestication. On dit d'une nouvelle pensée qu'elle risque d'être 'domestiquée' si elle est reçue dans le monde académique, ou encore si elle est diffusée couramment dans cet espace qu'on appelle 'le monde'. Les mouvements, dans les deux cas, sont inversés: dans le premier, il s'agit d'une entrée 'dans' l'institution à partir du dehors; dans le deuxième, d'une pénétration dans un monde représenté comme un dehors. Mais, dans un autre sens, cela revient au même: une originalité se perd en s'assimilant à ce dont on avait commencé par se différencier. Ce qui était, ou aurait pu être, authentique, sui generis, différent de la norme, est incorporé, assimilé, et perd tout pouvoir critique, toute différenciation.

La notion de domestication a été souvent utilisée, par exemple, en parlant du destin d'un féminisme 'en vrac', radical, qui aurait perdu ses capacités de lutte et de critique à partir du moment où il s'est loué un appartement ou un département à l'université. Ou pour parler de la déconstruction: à partir du moment, dit-on, où tous les départements de littérature comparée, d'anglais, etc., ont chacun leur petit théoricien, alors la théorie n'est plus qu'une spécialité parmi les autres, une nouvelle addition -- même pas un supplément, au sens de Derrida -- à la liste des options à choisir pour le 
succès professionnel. Ou encore: dès qu'un certain 'tout le monde', et pas uniquement un petit monde d'experts, a appris des slogans hors contexte sur la nouvelle théorie, ou dès que cette théorie est diffusée sous forme de 'introductory books' ou textes de présentation, on a également affaire à une domestication qui signifie alors la distorsion et la simplification de quelque chose de complexe.

Il y a deux sortes d'ironies, semble-t-il, dans ces utilisations de l'idée de domestication. L'une s'applique à la théorie en général, y compris la théorie féministe qui s'appuie sur la philosophie française. L'autre s'applique plus particulièrement au féminisme. Envisageons d'abord ce qui concerne les théories post-structuralistes. Car raconter une histoire où quelque chose -telle théorie -- est perçu d'abord comme originel, authentique, puis s'aliène, se perd, s'assimile, ne serait pas une histoire racontable pour la déconstruction pure et dure, si elle existe -- (mais on sait, bien sûr, que ni la pureté ni la dureté ne sont des concepts chers aux déconstructeurs pour qui le pur est toujours un petit peu impur, et le dur sans doute trop manifestement phallogocentrique).

Cette histoire d'une domestication de la théorie ne fonctionne pas justement parce qu'elle présuppose cela même que la déconstruction déconstruit, à savoir la notion d'une présence originaire et intégrale qui n'aurait qu'après coup, ou de façon contingente, différée de soi-même. Car la déconstruction, comme la théorie féministe ou toute autre théorie, est toujours déjà domestiquée, si par là on entend qu'elle n'est jamais sans rapport avec ce qu'elle vient penser ou déconstruire. Ce qui n'implique pas qu'on ne peut pas, ou qu'on ne devrait pas, essayer de marquer des distinctions entre les différentes conditions ou possibilités de domestication. Au contraire, du moment où on n'a plus recours à l'idée d'une pureté ou d'une présence originelles comme critères, il est d'autant plus important de faire de telles distinctions entre les utilisations -- les destins ou les domestications -possibles.

Voilà pour l'ironie qui se cache derrière cet usage de la notion de domestication par rapport à la théorie en général. Quant à la théorie féministe, l'ironie tient non pas à la structure narrative, mais à la signification du mot. Car la domestication, pour les femmes, n'est pas un thème quelconque. En fait, on pourrait dire que presque toute l'histoire de la pensée féministe occidentale des deux siècles passés est inséparable d'une répudiation de /pp. 17-18/ la domesticité, vue comme symbole ou symptôme privilégiés du refoulement et de l'oppression des femmes. Le domestique, en tant qu'espaces privé et familial, représente tout ce à quoi les femmes ont à échapper pour devenir libres et pour entrer dans la vie et l'espace publics. C'est là une tradition qui remonte à la fin du dix-huitième siècle -- je pense à Mary Wollstonecraft -- et qui persiste jusqu'aux grands textes du féminisme de notre siècle. Pensez au chapitre du Deuxième Sexe sur le mariage. La maison, pour Beauvoir, est une quasi-prison: là-dedans la 
femme n'arrive à rien faire, justement parce qu'elle ne cesse d'y répéter les mêmes tâches domestiques, des tâches sans fin et sans reste, sans produit qui dure.

On pourrait suggérer qu'il y a là des similarités par rapport aux recherches menées trente ans plus tard -- pendant les années 70 par Luce Irigaray et Julie Kristeva qui, toutes deux, se sont intéressées aux différents modèles du temps -- le linéaire, le cyclique surtout -- et leur rapport éventuel à la différence des sexes. Chez Irigaray, mais aussi chez Kristeva ( $>>$ Le temps des femmes $>>$ ), on retrouve des tentative différentes de proposer une sorte de revalorisation du temps féminin, c'est-à-dire d'un temps qui n'est pas téléologique et linéaire: le temps qui se répète, comme les saisons, les générations, la vie quotidienne.

Cette idée aurait pu conduire à une revalorisation du travail domestique et de la vie domestique toujours rejetés par les féministes. Mais il n'en est rien. Le temps non-linéaire, non-productif, demeure au niveau abstrait; il ne descend pas dans la maison, dans la vie concrète de tous les jours. Et si Kristeva et Irigaray parlent des réalités de la vie des femmes, c'est plutôt pour accepter, sans plus dire, le schéma classique du féminisme, style 'homologation' (pour parler comme Kristeva): bien sûr que les femmes devraient avoir le droit d'entrer dans le monde public représenté comme l'espace d'une liberté exclue de la maison. Je ne dis pas ça pour célébrer la vie de ménagère, mais plutôt pour signaler à la fois la persistance et l'occlusion du thème de la domestication si intimement lié aux questions féministes.

Pour conclure, dans le même sens, je vais analyser les enjeux d'un livre beaucoup plus récent que ceux dont j'ai parlé jusqu'à présent: Gender Trouble de Judith Butler, qui a été publié en 1990.[2] (Je n'ose pas risquer une traduction du titre -- j'ai l'impression que j'aurais du trouble avec le mot gender, que je m'emmêlerais très vite dans des histoires de belle-mère...) C'est un livre qui a eu beaucoup d'influence aux États-Unis, en partie parce qu'il a semblé offrir un moyen de sortir des impasses du débat sur l'essentialism. Butler s'appuie surtout sur Foucault, et aussi sur Derrida: de Foucault, elle retient l'idée d'une production continue de sujets qui sont 'construits', constructed, de part en part; de Derrida, l'idée d'une copie sans original. L'original - -- en matière de gender, comme ailleurs -- ne serait qu'un effet rétroactif produit dans un deuxième temps où la copie paraît comme secondaire. Bien sûr, le partage Foucault/Derrida n'est pas aussi simple. Et ce que je n'ose pas appeler l'originalité de Butler (mais quand même...) consiste néanmoins en ce qu'elle produit du nouveau. Étant donné une situation générale où tout est déjà construit, secondaire, et où il n'y a aucun fond de nature, Butler en propose un pastiche généralisé. On ne peut pas sortir du monde donné pour en faire la critique de l'extérieur. On ne 
peut que jouer avec ce qui est, ce qui se fait, pour en montrer ou en exposer la contingence.

Avant de passer à des considérations plus générales, je veux m'arrêter un instant sur le thème de la maison. Car chez Butler aussi, on voit apparaître subrepticement une certaine idée de la domestication, presque cachée par l'usage d'une métaphore habituelle:

The complexity of gender requires an interdisciplinary and postdisciplinary set of discourses in order to resist the domestication of gender studies or women's studies within the academy and to radicalize the notion of feminist critique. (xiii)

Ici, la domestication est définie tout simplement comme ce à quoi il faut résister: c'est le contraire de la radicalisation et ça demeure quelque chose de simple, versus 'the complexity of gender'. Ou encore:

Parody by itself is not subversive, and there must be a way to understand what makes certain kinds of parodic repetitions effectively disruptive, truly troubling, and which repetitions become domesticated and recirculated as instruments of cultural hegemony.(139)

Dans les deux cas, le mot domestication représente la normalisation, la perte de ce qui aurait pu être vraiment subversif -- 'effectively disruptive, truly troubling'. La domestication vient à s'identifier ici avec un instrument de l'hégémonie culturelle, rien de moins.

Ce qui m'intéresse dans ces citations, c'est qu'elles reviennent justement au type d'argument qui est rejeté par Butler avec tant de force et de précision. Quand elle invoque la domestication, une opposition claire entre ce qui est primaire et ce qui est secondaire reste en place, sans être mise en question. Encore moins, s'interroge-t-elle sur le contenu thématique du terme.

Je crois que le cas de la domestication, par lequel Butler revient justement à la structure de pensée qu'elle est en train de critiquer, n'est pas tout à fait unique ou excentrique dans son texte. Car son argument, qui se présente comme une critique de la notion de fond et une critique des divisions binaires, en matière de sexe et autre, dépend lui-même d'un fond caché -- le fond du non-fond -- et aussi, il me semble, d'oppositions binaires. Par exemple: 
If gender attributes [...] are not expressive but performative, then these attributes effectively constitute the identity they are said to reveal. The distinction between expression and performativeness is crucial. If gender attributes and acts, the various ways in which a body shows or produces its cultural signification, are performative, then there is no preexisting identity by which an act or attribute might be measured; there would be no true or false, real or distorted acts of gender, and the postulation of a true gender identity would be revealed as a regulatory fiction. (141)

Notons d'abord l'opposition manifeste entre expressive et performative. Pour Butler, l'expressif est négatif. Il tient à l'idée d'une essence primaire et originelle qui serait par la suite exprimée, tandis que le performatif serait la productivité mais aussi l'état des choses. Ce que Butler appelle la fiction régulative des essences, des identités stables, est une fiction produite ou acted out, au sens fort.

Dans le passage que je viens de citer, on peut voir à quel point cette distinction entre l'expression et la performance, ou entre l'essence et la production, est fondamentale. Mais on peut voir aussi que l'affirmation de la performativité dépend, elle aussi, d'une distinction du primaire et du secondaire qui est strictement identique à celle critiquée par l'auteur. Elle dit, en effet: non, ce n'est pas l'expression qui est primaire, c'est la performance; non, ce n'est pas le primaire qui est primaire, c'est le secondaire: le primaire vient après, en deuxième lieu.

On y reconnaît aussi une rhétorique de la révélation, du dévoilement, qui est tout à fait classique. Cette rhétorique est d'abord identifiée avec la structure expressive que Butler est en train de critiquer: <<these attributes effectively constitute the identity they are said to express or reveal $>>$. Mais deux phrases plus loin, elle poursuit: $<<$ the postulation of a true gender identity would be revealed as a regulatory fiction $>>$. Exposé, tout de même, d'une vérité du manque de vérité.

Il n'est pas difficile de déceler chez Butler toutes sortes de valeurs implicites ou parfois explicites, qui auraient pu, à leur tour, subir le genre -- pas le gender -- de critique qu'elle pratique à l'égard des notions d'essence ou d'identité sexuelle originaire. Quelles sont les valeurs positives pour Butler? Le performatif contre l'expression, on l'a déjà vu. À celle-ci se lient: la pratique de la signification contre le sens existant, la mobilité contre la solidité ou la fixité, la production continue contre le produit, les effets contre les causes qui ne sont établies telles que rétroactivement. 
Mais ces distinctions de valeurs fonctionnent aussi, pour Butler, comme descriptions de la réalité, ou plutôt de ses conditions de production ou de construction. Comme chez Foucault, il y a pour Butler des instances de législation ou de prescription qui ont des effets, qui produisent à chaque moment toutes les formes possibles et provisoires d'être. C'est de cette même activité, de ce même agencement, que Butler sollicite, invite l'imitation. Car si on ne peut pas résister au nom d'une essence ou d'une vérité quelconques, on peut et on doit quand même essayer d'opérer une sorte d'opposition à partir du dedans, où on s'expose en simulant justement les opérations de cette même loi. On joue un rôle en sachant que c'est un rôle, en connaissance de cause -- ou plutôt, pour Butler, en connaissance de cause-produite-par-son-effet:

As the effects of a subtle and politically enforced performativity, gender is an "act," as it were, that is open to splittings, self-parody, self-criticism, and those hyperbolic exhibitions of "the natural" that, in their very exaggeration, reveal its fundamentally phantasmatic status. (146-7)

Encore ce mot 'reveal' -- ici la révélation d'une subtilité, $<<$ a subtle and politically enforced performativity >>; ailleurs, Butler se sert du mot insidious:

Ontology is, thus, not a foundation, but a normative injunction that operates insidiously by installing itself into political discourse as its necessary ground.(148)

Des deux côtés, donc, on triche: du côté des injonctions normatives, on est subtil, insidieux; du côté de la résistance qui n'en est pas une, on fait semblant d'imiter, on imite jusqu'à l'excès. Comme le reconnaît Butler ellemême, je crois, on ne saurait pas trop distinguer entre les deux sortes d'opérations, sauf par une décision arbitraire (qui s'effectuerait contre sa théorie) en faisant appel aux intentions, aux pseudo-causes donc et non pas aux effets. Il est vrai aussi que l'idée de la subjectivité comme performance, et non comme nature, n'est pas forcément radicale: pensez, par exemple, à la sociologie la plus normale, sinon normative, où on parle couramment des rôles appris ou adoptés par les sujets comme personnages dans une famille, au travail, etc.

Je passe par ce long trajet d'une analyse textuelle pour en arriver, j'espère, à deux points culminants -- sinon à trois derniers points. Premièrement, je voudrais signaler la continuation, sous cette nouvelle forme, des enjeux représentés par l'opposition féminisme/women's studies. Dans cette opposition, les women's studies étaient représentées comme contenu, 
essence, fixité, institution; le féminisme comme subversion, opération aux marges, mobilité. Chez Butler, les termes de la valorisation n'ont pas changé.

Deuxième point: en lisant et relisant Butler, j'ai été frappée par l'insistance d'un mot dont elle se sert tout le temps, et toujours avec une connotation positive, celui de prolifération. Voici quelques citations, tirées au hasard des dernières pages:

The task is not whether to repeat, but how to repeat, or, indeed, to repeat and, through a radical proliferation of gender, to displace the very gender norms that enable the repetition itself.(148)

The loss of gender norms would have the effect of proliferating gender configurations, destabilizing substantive identity.(147)

Cultural configurations of sex and gender might then proliferate or, rather, their present proliferation might then become articulable within the discourses that establish intelligible cultural life, confounding the very binarism of sex and exposing its fundamental unnaturalness.(149)

Dans cette dernière citation, un parallèle est reconnu entre les pratiques qui renforcent les normes et celles qui les subver- tissent: dans les deux cas, il y a prolifération. D'où la nécessité d'essayer de proliférer dans le bon sens, en parodiant par exemple.

Mais en quoi consiste cette prolifération qu'on rencontre partout? Comme la domestication, c'est un mot à cinq syllabes, très satisfaisant à prononcer, qui se glisse subrepticement, sinon insidieusement, dans le discours sans jamais se montrer autrement que naturel, sans jamais être remis en question. Il existe néanmoins un autre type de contexte où ce mot est très répandu, où il prolifère: je pense au discours sur les armes nucléaires. Là, évidemment, la connotation du mot est carrément menaçante.

Étymologiquement, la prolifération est très simple: c'est l'idée de reproduction, prolem ferre, qui donne la même racine au prolétariat, ces êtres qui n'existent que pour se reproduire comme des lapins. Ce que Butler propose donc, c'est la reproduction aveugle, sans arrière-pensée et sans planning, familial ou autre. Pas de contrôle, ça se fait tout seul et ça se fait quoi qu'on en pense, qu'on vous y pousse ou pas. Il n'y a plus de sujet, que 
ce soit un sujet conforme aux lois ou un sujet qui les parodie; il n'y a que cet automatisme animal d'un reproduire sans cesse.

Proliférons donc, pourquoi pas... mais je crois que ce spectacle (nous sommes des voyeurs, après tout, pas si aveugles que ça) nous offre peut-être l'indication d'un problème dans le schéma de Butler. En dernière instance, la question du sujet choisissant, au singulier ou au pluriel, est esquivée: il n'y a que cette activité reproductrice qui parfois se parodie (ou se pastiche) ellemême. Il existe une sorte de bonne volonté quelque part -- une volonté de parodier, de changer -- à qui revient la tâche de trancher entre la bonne et la mauvaise prolifération, la normative et la subversive. C'est le bon vieux sujet moral, mais d'où vient-il?

Troisième et dernier point, qui n'est pas sans rapport avec le deuxième. Le livre de Judith Butler est souvent marqué par une insistance portant sur l'importance et l'efficacité du rire -- et cette notion du rire s'appuie ellemême un peu sur le fameux essai d'Hélène Cixous, $<<$ Le rire de la Méduse $>>$. Elle écrit par exemple:

The loss of the sense of "the normal," however, can be its own occasion for laughter, especially when "the normal," "the original" is revealed [encore ce 'révéler'!] to be a copy, and an inevitably failed one, an ideal that no one can embody. In this sense, laughter emerges in the realization that all along the original was derived.(138-9)

Le livre de Butler (et je crois que je ne suis pas seule à le penser) n'est pas un livre qui fait rire. Ce n'est pas une parodie du style ou des règles de l'argument philosophique; c'est une série de critiques faites selon toutes les conventions du genre (sinon du gender), sans plaisanterie, sans glissements de langue. Je crois que ce décalage entre le thème choisi par Butler -- la parodie comme seule possibilité de critique -- et son style archi-sérieux, très respectueux des lois du genre donne à penser (mais peut-être aussi à rire).

Il s'agit d'une ironie supplémentaire qui suscite le rire, sans doute, mais qui fait aussi ressortir une question très sérieuse sur le style des écrits féministes, ou des féministes. Mises à part les questions de contenu ou de matière, que devrait faire un texte féministe, quel effet devrait-il essayer de produire?

La forme de ma question est trop normative. En vérité, je ne recherche pas de prescription globale sur ce sujet. Mais je crois que c'est une question très importante surtout quand on veut parler, dans les termes de Butler, du 
discours comme productif. Je suggère donc un titre supplémentaire pour ce livre: non pas tellement Gender Trouble, mais plutôt Genre trouble.

\section{Rachel Bowlby}

\section{Department of English}

Sussex University

Surface Page d'Acceuil/Home Page

[1]Julia Kristeva, 'Women's Time', trans. Alice Jardine and Harry Blake, in Signs: A Journal of Women in Culture and Society, 7:1 (Fall 1981), 13-35.

[2]Judith Butler, Gender Trouble: Feminism and the Subversion of Identity (New York: Routledge, 1990). 